\title{
Ceramide and mitochondrial function in aging oocytes: joggling a new hypothesis and old players
}

\author{
Loro L Kujjo ${ }^{1,2}$ and Gloria I Perez ${ }^{2,3}$ \\ ${ }^{1}$ Department of Human Anatomy, Michigan State University, A502B East Fee Hall, East Lansing, Michigan, USA, \\ ${ }^{2}$ Department of Physiology, Michigan State University, 4173 BPS, East Lansing, Michigan 48824, USA and ${ }^{3}$ LARCel, \\ Programa Andaluz de Terapia Celular y Medicina Regenerativa, Sevilla, Spain
}

Correspondence should be addressed to G I Perez at Department of Physiology, Michigan State University; Email: perezg@msu.edu

\begin{abstract}
Maternal aging adversely affects oocyte quality (function and developmental potential) and consequently lowers pregnancy rates while increasing spontaneous abortions. Substantial evidence, especially from egg donation studies, implicates the decreased quality of an aging oocyte as a major factor in the etiology of female infertility. Nevertheless, the cellular and molecular mechanisms responsible for the decreased oocyte quality with advanced maternal aging are not fully characterized. Herein we present information in the published literature and our own data to support the hypothesis that during aging induced decreases in mitochondrial ceramide levels and associated alterations in mitochondrial structure and function are prominent elements contributing to reduced oocyte quality. Hence, by examining the molecular determinants that underlie impairments in oocyte mitochondria, we expect to sieve to a better understanding of the mechanistic anatomy of oocyte aging.

Reproduction (2012) 1431-10
\end{abstract}

\section{Maternal age and fertility}

Female fecundity (the ability to produce offspring) reaches its peak at 30 years of age, then declines abruptly and is lost by $\sim 50$ years of age at the onset of menopause (Faddy 2000, Gougeon 2005). This phenomenon is due to a combination of factors, but in general, subfertility in older women is primarily related to: poor quality of aging oocytes, reflecting chromosomal, morphological, and functional abnormalities (Beemsterboer et al. 2006, Hunt \& Hassold 2008, Broekmans et al. 2009, Perheentupa \& Huhtaniemi 2009); decreased ovarian reserve, marked by fewer oocytes (Richardson et al. 1987, Ames et al. 1995, Broekmans et al. 2009, Perheentupa \& Huhtaniemi 2009); and an altered hormonal environment resulting in ovulatory dysfunction (Broekmans et al. 2009). Only about $5 \%$ of women with diminished ovarian reserve presently achieve pregnancy, despite use of ovulation inducing agents (Gougeon 2005, Braveman 2006, Habbema et al. 2009). In the last 30 years a worldwide trend by women to delay childbearing until onset of the decline in fecundity (late 30 s and 40s) has increased the risk of infertility (Ottolenghi et al. 2004). Assisted reproductive technologies (ART) have compensated for the decreased natural fertility, but only to a limited extent (Braveman 2006, Habbema et al. 2009), leaving many couples childless despite prolonged and demanding therapies for infertility.

Owing to lack of knowledge, the mechanisms behind the gradual decline of the follicle pool and the reduced oocyte quality are far from being fully understood. According to Hunt \& Hassold (2008), female fertility is influenced by a complex series of events that occur at any of the three critical stages of oocyte development, identified as: the initiation of meiosis in the fetal gonad; formation of primordial follicles during late gestation and the perinatal period; and oocyte growth and maturation taking place in the adult. Thus, it seems increasingly likely that age-related changes affecting the quality of the oocyte reflect a complex interplay of events involving both nuclear and cytoplasmic components.

Since age is highly negatively correlated with oocyte quality, advance maternal age is the most prominent impediment to a successful assisted reproductive program Janny \& Menezo 1996, Hunt \& Hassold 2008, Habbema et al. 2009). The evidence for impact of age is apparent in the increased percentages of embryo fragmentation during early cleavage stages in embryos from older women (Jurisicova et al. 2006), and by an elevated cell death index in blastocysts from aged mice (Keefe et al. 1995, Acton et al. 2004).

A mathematical model of death rates in human preimplantation embryos suggests that the factors 
predisposing an embryo to developmental arrest are determined at the zygote stage or earlier (Hardy et al. 2001). The initial stages of embryo development are sustained by maternally provided transcripts and proteins accumulated in the oocyte during oogenesis (Schultz \& Heyner 1992, Minami et al. 2007, Tang et al. 2007). These factors are crucial for early developmental events in embryos, including axis formation, cell fate determination, and activation of the embryonic genome. Furthermore, postfertilization, the oocyte cytoplasm is solely responsible for remodeling of the incorporated paternal chromatin and plays a role in epigenetic modifications of the newly formed embryonic nucleus (Torres-Padilla et al. 2006, Yoshida et al. 2007). Since factors from the oocyte cytoplasm and products of its gene expression control many processes central to the early development of the whole organism, it is not surprising, therefore, that a decline in oocyte quality has a profound impact on the developmental competence of the embryo and contributes to the high incidence of embryonic wastage observed during IVF procedures in ART clinics (Jurisicova \& Acton 2004).

\section{Mitochondria in germ cells}

Mitochondria are the most prominent cell organelles in oocytes, and they represent one of the most important maternal contributions to early embryogenesis (Van Blerkom 2004, 2011, Van Blerkom et al. 2006, Dumollard et al. 2007, Eichenlaub-Ritter et al. 2011). Owing to their role in the production of cellular energy as well as in the control of cell death, these organelles are at the center of death and life decisions in most cells, including the oocyte (Perez et al. 2000, Wang 2001, Danial \& Korsmeyer 2004). Moreover, mitochondrial activity appears to be essential for normal spindle formation and chromosome segregation (EichenlaubRitter et al. 2004). However, compared with the other cell types in the body, germ cells' mitochondria are unique. For example, in comparison to those in somatic cells, oocyte mitochondria have been described as morphologically primitive or immature, due to the fact that they are smaller and possess less complex internal structures (Dumollard et al. 2007).

The number of cristae in a mitochondrion has been directly correlated to the level of ATP production (Jansen \& de Boer 1998). As such, the germ cell mitochondria with their limited number of cristae and denser matrices have diminished ability to generate ATP. This has been demonstrated in human oocytes by Van Blerkom et al. (1995). Recently, several studies on the metabolic activity of oocytes and early embryos concluded that, although their mitochondria have low activity, they are constitutively active; and that maintenance of that low-level activity is necessary and sufficient for ongoing development (Cummins 2004a, b, Van Blerkom 2004, 2011, Van Blerkom et al. 2006, Dumollard et al. 2007).
Nevertheless, in aged mouse oocytes we have recently found that the number of mitochondrial cristae is even lower compared with younger oocytes, which may compromise development. It should be noted that mitochondria in a zygote are maternally inherited, the few paternal mitochondria that enter the oocyte during fertilization are targeted to ubiquitin-dependent proteolysis (Giles et al. 1980, Cummins 2000, 2002, Sutovsky et al. 2000), or diluted out during sequential cleavage (Cummins 2000). Since the mitochondria in oocytes have a limited number of replication cycles before the blastocyst stage (McConnell \& Petrie 2004), the developing embryo is inevitably designed to depend entirely on the population of mitochondria present at the time of ovulation. Moreover, in contrast to most cells, oocytes and cleavage stage embryos rely on mitochondrial ATP alone since glycolysis is pretty much switched off.

It is reported that deletions in mitochondrial DNA (mtDNA) are significantly higher in oocytes than in embryos, strongly suggesting that mtDNA integrity plays a role in determining the fertilizability of oocytes (Brenner et al. 1998, Barritt et al. 1999). Evidence that mitochondria directly determine the fate and quality of oocytes and embryos has been demonstrated by us and others in studies where microinjection of pure mitochondria or mitochondria-enriched cytoplasts into mouse oocytes reduced apoptotic rates (Perez et al. 2000) and increased ATP production (Van Blerkom et al. 1998). Additional support is provided by the fact that ooplasm transfer to poor quality recipient oocytes in ART clinics decreased oocyte and embryo fragmentation rates, improved embryo development and led to the birth of at least 30 children worldwide (Cohen et al. 1998).

Therefore, as in any other cell, it is expected that adequate generation of energy within oocytes will be of primary significance for processes such as oocyte maturation, chromosome segregation, and developmental capacity (Eichenlaub-Ritter et al. 2010). Any changes in activity of mitochondria (e.g. redox homeostasis) and in their morphology, as those seen during aging, are thus expected to be crucial for fertility. Ageassociated mitochondrial damage is anticipated to be most critical for stages with higher energy demands such as oocyte maturation and early embryo development right up to the time of zygotic gene activation.

\section{Mitochondria and oocyte aging}

Mitochondrial dysfunction has been implicated in both general body aging (Ames et al. 1995, Sastre et al. 2002) and aging of female reproductive tissues (Janny \& Menezo 1996, Jansen \& de Boer 1998, Kirkwood 1998, Ruman et al. 2003, Tarlatzis \& Zepiridis 2003, Ottolenghi et al. 2004, Gougeon 2005). Our most recent observations demonstrate that, both function and morphology of mitochondria are impaired in oocytes from older mice (GI Perez \& LL Kujjo 2011, unpublished 
observation). And hence, considering the importance of mitochondrial function in the oocyte, it is not surprising that oocyte quality decreases with aging (Ruman et al. 2003, Tarlatzis \& Zepiridis 2003, Ottolenghi et al. 2004, Gougeon 2005). This in turn may translate into a fall in the success rates of IVF cases, partially due to increases in oocyte mitochondrial defects. Evidence for this is further provided by the correlated increase of maternal age with: increase in the rate of DNA mutations in oocyte mitochondria (Keefe et al. 1995); decrease in mitochondrial metabolic activity (Wilding et al. 2001); inefficiency in mitochondrial ATP production (Van Blerkom et al. 1995); and changes in mitochondrial calcium homeostasis (Van Blerkom 2011).

In elegant experiments using mouse oocytes as a model of mitochondrial dysfunction, Thouas et al. (2004, 2005 , 2006) demonstrated that sensitivity to mitochondrial injury is developmentally regulated and increases with age. For example, when young oocytes were subjected to low degrees of mitochondrial injury by exposing them to photosensitization, further development to blastocyst in vitrowas not affected. By comparison, in vitro development of preimplantation embryos from aged oocytes was more sensitive to equivalent mitochondrial damage. The authors concluded that agerelated mitochondrial energy deficiency might account for the differences observed, and suggested a possible direct linkage of delayed developmental effects to mitochondrial deficiencies caused by advanced aging (Thouas et al. 2005). These data are in agreement with those from human oocytes, showing that decreases in activity of mitochondria derived from oocytes of older women are associated with lower embryonic development and low pregnancy rates compared with oocytes obtained from younger women (Van Blerkom et al. 1995).

In addition to generalized aging of oocyte cytoplasm, increase in dysfunctions of mitochondria with advanced age might also contribute to nuclear genome instability in oocytes, as reported for other cells (Veatch et al. 2009). As such, age-related increases in aneuploidy in oocytes might be direct or indirect consequences of decreased mitochondrial activity. For example as described in recent publications (Cukurcam et al. 2007, Eichenlaub-Ritter et al. 2010), occurrence of spindle aberrations attributed to insufficient energy supply, and/or shifts in redox regulation, might influence enzyme activities and cause loss of chromosome cohesion, or chromosome integrity and stability, especially during early embryogenesis. Thus, there is sufficient evidence that with advanced age mitochondrial pathophysiology contributes to decreased female fertility. However, the causative factors leading to aging-related changes in mitochondrial structure and function for the most part remain unknown.

Although age-related mutations in oocyte mtDNA affect oocyte quality, it is anticipated that those effects would be subtle, since only a fraction of the numerous mitochondria present in the germ cells would be affected (Eichenlaub-Ritter et al. 2011). On the other hand, we hypothesize that age-related alterations in mitochondrial lipids or any other global structural mitochondrial damage are expected to have a more pronounced impact on overall mitochondrial activity because they affect all mitochondria.

\section{Ceramide and oocyte aging}

Considering the preceding discussion on the impact of mitochondria on oocyte quality, we hypothesize that: during aging, a dysregulation in the intracellular transport and/or synthesis of ceramide (a bioactive lipid) occurs, and that this prevents ceramide from reaching the normal levels in mitochondria. This lipid imbalance leads to less functional mitochondria and a negative effect on oocyte quality.

Ceramide is the basic structural component of many lipids known as sphingolipids. In this central role, ceramide is utilized for the synthesis of other bioactive sphingolipids, including sphingosine-1-phosphate, and sphingosine (SPH), and the glycosphingolipids hexosylceramide and lactosylceramide (Hannun \& Obeid 2011).

Sphingolipids, specifically ceramide, as well as mitochondria, are implicated in both development and aging at the organismal (Cutler \& Mattson 2001) and the tissue level (Lightle et al. 2000). Currently, 200 distinct ceramide species are known to exist in mammalian cells (Hannun \& Obeid 2011). These ceramides are synthesized in a combinatorial fashion, with distinct enzymes responsible for the specific modifications (Gault et al. 2010). As shown by Hannun \& Obeid (2008) ceramides can be generated through the action of sphingomyelinase (SMase) or via the de novo synthetic pathway through the action of ceramide synthases (CerSs). Ceramides generated via SM hydrolysis can be further hydrolyzed by ceramidases (CDases) to form $\mathrm{SPH}$, which can then be reacylated via the action of CerS (also known as LASS) to regenerate ceramide species. These multiple pathways of ceramide generation led to the hypothesis that individual ceramide molecular species are regulated by specific biochemical pathways in distinct subcellular compartments and execute distinct functions (Hannun \& Obeid 2011, Novgorodov et al. 2011).

Mitochondria are important intracellular compartments of sphingolipid metabolism (Novgorodov \& Gudz 2009), and several sphingolipid-metabolizing enzymes were found to be associated with mitochondria; so far the list includes neutral ceramidase (EI Bawab et al. 2000), novel neutral SMase (Wu et al. 2010, Clarke et al. 2011), and (dihydro) CerS, a key enzyme in de novo ceramide synthesis (Bionda et al. 2004, Yu et al. 2007). The existence of these compartment-specific pathways clearly suggests high specialization of these pathways, which in turn suggests specific mechanisms of 
regulation and, equally as likely, distinct functions and mechanisms of action of their lipid products.

Ceramide signaling involves a complex network of molecules and subcellular organelles all implicated in a range of cellular processes such as necrosis (Hetz et al. 2002), survival and proliferation (Adam et al. 2002), differentiation (Okazaki et al. 1990), and aging (Venable et al. 2006). The involvement of sphingolipids in aging is not new; after all, there is considerable evidence linking sphingolipid genes with lifespan. For example, deletion of the longevity assurance gene (LAG1), one of the first genes implicated in yeast aging ( $\mathrm{D}^{\prime}$ Mello et al. 1994) was shown to extend lifespan. Interestingly, this gene was subsequently discovered to encode a CerS (Guillas et al. 2001, Schorling et al. 2001). Furthermore, some pathways of sphingolipid metabolism have been shown to play an important role in determining Drosophila lifespan (Rao et al. 2007, Yang et al. 2010). Recently, Yang et al. (2010) reported that the Drosophila alkaline ceramidase (catalyzes breakdown of ceramide into SPH and fatty acids) plays an important role in Drosophila development and longevity; apparently, the mutation of alkaline ceramidase significantly increases mean and maximum lifespan. Finally, mutations of the ceramide transfer protein (CERT) in flies caused enhanced oxidative damage and dramatically reduced their lifespans (Rao et al. 2007). By virtue of its function, CERT regulates cellular ceramide levels by transporting ceramide from the endoplasmic reticulum (ER) to the Golgi where it is used specifically for SM synthesis (Hanada et al. 2007). Taken together, these studies support the role of sphingolipids in determining lifespan, and as such are important regulators of the aging process.

It is perhaps not surprising that we recently identified ceramide as one of the key molecules signaling the accelerated incidence of apoptosis in oocytes of aged female mice (Perez et al. 2005). This finding highlights a novel role for the intercellular trafficking of ceramide as a key step in this process of accelerated oocyte death. Therefore, we believe that this novel role of ceramide is associated with the aging-related decline of ovarian function (Richardson et al. 1987, Faddy et al. 1992). Our conclusion is supported by the fact that ceramide levels have been observed to increase during the years immediately preceding menopause (Diatlovitskaia et al. 1995), a phase in a woman's life when the endowed pool of germ cells (oocytes) has been nearly exhausted (Richardson et al. 1987).

We demonstrated that in aged mice the levels of ceramide increase in the cumulus cells but decrease in the oocyte. This chronic depletion of oocyte ceramide is a prerequisite that sets up the oocyte to become sensitive to ceramide spikes released by the cumulus cells and translocated via gap junction-dependent communication (Perez et al. 2005). So, in the aging oocyte we have delineated two patterns of ceramide concentrations: one dependent on what we call 'precumulus release', characterized by lower levels of ceramide, such as the ones we previously reported (Perez et al. 2005); this occurs around 9 months of age in ICR mice. The second concentration pattern is sustained by 'post-cumulus release'; it occurs somewhere after 12 months of age, being characterized by high levels of ceramide, and is responsible for accelerated oocyte death with age. Considering the fact that the accelerated rate of oocyte depletion from the human ovaries coincides with the time when ceramide levels increase, it is logical to speculate that the events we observed in the aging mice also underlie similar mechanisms in aging human oocytes. Nevertheless, so far no data have been published to indicate any association between human maternal age and the lipid content of oocytes.

In further studies we also discovered that signaling of survival and death pathways in the oocyte are influenced by the spatial locations of ceramide pools (Perez et al. 2005). In oocytes from young mice, exogenous ceramide rapidly accumulates in mitochondria and prevents oocyte apoptosis. In contrast, in oocytes from aged mice, exogenous ceramide does not reach the mitochondria but accumulates in the cytoplasm (possibly in the ER) and triggers cell death (Fig. 1; GI Perez 2011, unpublished observation). The emerging view is that distinct ceramides in independent compartments serve as local centers of sphingolipid metabolism (Hannun \& Obeid 2011). In turn, protein-mediated transfer and vesicular transport of sphingolipids then serve to connect these various centers of activity. It is quite unlikely that ceramide formed in the lysosomes by the action of acid SMase would exert the same specific effects as ceramide formed in the mitochondria or the plasma membrane by the regulation of CDases or neutral SMase. Therefore, mechanistic studies on ceramide function and regulation should focus on specific pathways of formation. Three recent reviews have highlighted the physicochemical dynamics of ceramide signaling, and stressed the need to pinpoint the specific membrane ceramides associate with (van Blitterswijk et al. 2001, Hannun \& Obeid 2008, 2011). Our laboratory is currently monitoring and localizing the cytoplasmic pools of ceramide in oocytes and their relationship to age, oocyte quality, and developmental potential. Currently, it is difficult to predict whether an observed change in ceramide will lead to apoptosis or ER stress or senescence. According to the new hypothesis, defining the pathway regulating ceramide, where in the cell this occurs, and what specific species of ceramide are involved will result in a much more robust understanding of that specific pathway of cell regulation and in predicting its function.

\section{Ceramide transport protein and aging}

In the cell, several organelles are responsible for the synthesis of ceramide (see recent review by 

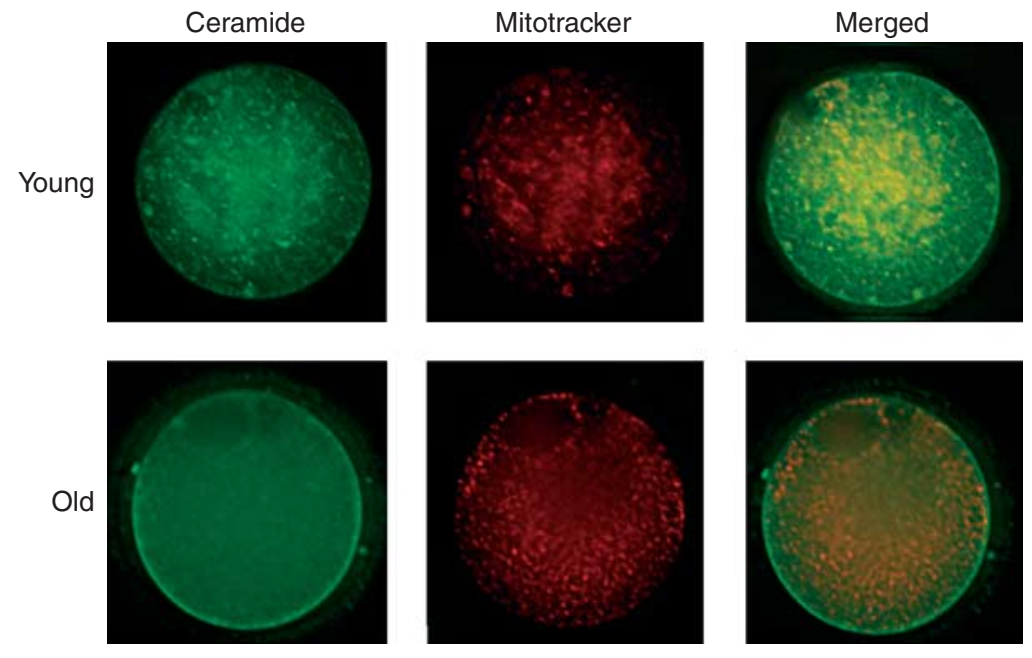

Figure 1 Exogenous ceramide localizes primarily to the mitochondria in young oocytes but not in old oocytes. Colocalization of ceramide and mitochondria in young oocytes is depicted in the merged image (yellow color). Methods: mature oocytes were cultured in HTF medium supplemented with $0.5 \% \mathrm{BSA}$ at $37^{\circ} \mathrm{C}$ in the presence of fluorescent ceramide $(60 \mu \mathrm{M})$ for $1 \mathrm{~h}$. After $30 \mathrm{~min}$, mitotracker red was added (250 nM) for an additional $30 \mathrm{~min}$. Oocytes were washed in excess of media and visualized live using deconvolution microscopy.
Hannun \& Obeid (2011)). The pathway for the de novo synthesis of ceramide is initiated in the ER and continues in the Golgi. In addition, ceramide synthesis also takes place in the plasma membrane, and most probably in the mitochondria as well. Some ceramide molecules are highly hydrophobic and therefore, they need to be generated in close proximity to their site of action by the activity of enzymes located near by. In the case where transport between organelles is required, specific sphingolipid-binding transporter proteins are involved (Futerman \& Riezman 2005, Hannun \& Obeid 2011). To date, CERT is the only known specific ceramide transport protein. This protein was identified by Hanada (2006) and works in the non-vesicular transport of ceramide from the ER to the Golgi apparatus. CERT is also known as the Goodpasture antigen-binding protein (Hanada et al. 2003, 2007, Hanada 2010, Mencarelli et al. 2010).

The biochemical and physicochemical aspects of CERT function have been extensively studied by Hanada et al. (Hanada et al. 2003, 2007, Kumagai et al. 2005, Hanada 2006, Kawano et al. 2006) by in vitro models. But, it was not until recently that the function of CERT was studied in a relevant physiological model, when Rao et al. (2007) demonstrated that CERT function is essential for normal oxidative stress response and lifespan in Drosophila. Recently, the same group (Wang et al. 2009) demonstrated that CERT is essential for mouse development and embryonic survival. Interestingly, these authors also found that CERT is critical for mitochondrial integrity.

Of particular interest is the fact that the majority of the phenotypic changes in the CERT mutant flies have striking similarities with the changes we and others have observed in the aged (human and mouse) oocyte. Specifically, the mutant flies: 1) are susceptible to reactive oxygen species; 2) develop metabolic imbalance including decreased ATP levels; 3) exhibit a 70\% decrease in ceramide levels; and 4) die early in life. The findings in aged oocytes include: higher susceptibility to reactive oxygen species (ROS; Thouas et al. 2005); reduced ATP levels (Van Blerkom et al. 1995); and lowered levels of total ceramide (Perez et al. 2005) that precede an increased rate of apoptosis. Since there are striking similarities between changes observed in the mutant flies lacking CERT and the changes observed in the aging oocytes, we hypothesized that in the aged oocyte CERT is downregulated, with consequential compromise of the oocyte developmental potential. A prediction that we recently confirmed (GI Perez \& LL Kujjo 2011, unpublished observation).

Interestingly, it has been recently shown that the intermembrane ceramide transport catalyzed by CERT is sensitive to fluidity of the lipid environment (Tuuf et al. 2011). Therefore, if ceramide is in a tightly packed environment, the CERT transfer activity is markedly reduced. On the other hand, the ceramides in more fluid membranes are more available for CERT-mediated transfer. CERT also favors membranes that contain phosphatidylinositol 4-monophosphate (PI4P; Tuuf et al. 2011). Of interest is that, among the subcellular changes associated with advanced age can be discerned increases in membrane rigidity (Pepe 2005) and decreases in PI4P content of organelle membranes such as Golgi and mitochondria (Tran et al. 1993). Therefore, it would not be totally surprising to find that the transport of ceramide might also be impaired in aged oocytes.

\section{Hypothetical model of ceramide trafficking in oocytes}

The current literature and our data (published and unpublished) provide support for the involvement of ceramide in the age-dependent changes of oocyte mitochondrial structure and/or function, and led us to our hypothetical model (Fig. 2). The model posits that 


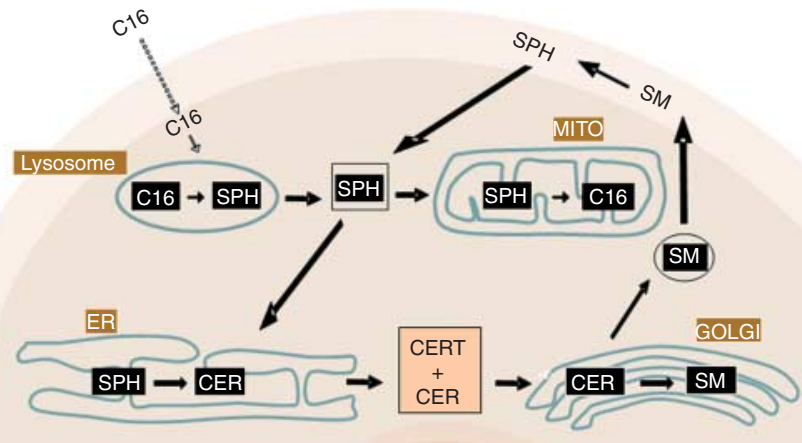

Nucleus
Figure 2 Hypothetical model of ceramide trafficking in oocytes. We hypothesize that poor quality of old oocytes results from ceramide accumulating in the ER as a consequence of disabled ceramide transport to the Golgi, due to downregulation of CERT during aging. This indirectly reduces mitochondrial levels of ceramide, because the cell fails to provide sufficient sphingosine for the synthesis of mitochondrial ceramide. SPH, sphingosine; SM, sphingomyelin; C16, C16-ceramide; CER, ceramide; CERT, ceramide transport protein. downregulation of CERT with advanced aging (GI Perez \& LL Kujjo 2011, unpublished observation) is the modular response responsible for the changes in the organellar levels of ceramide.

We propose that under normal conditions ceramide (C16-ceramide) originating from the cumulus cells reaches the lysosomal compartment of an oocyte where it is hydrolyzed to $\mathrm{SPH}$. SPH is then transported preferentially to the ER, but some little trafficking is directed to the mitochondria. In the ER and mitochondria (MITO) de novo synthesis of ceramide (CER) occurs. The ceramide formed in the ER is transported to the Golgi, which is the site of synthesis of SM. The transport of CER to the Golgi occurs through the action of the transfer protein CERT that specifically delivers CER for SM synthesis. SM is delivered to the plasma membrane where it is metabolized to other bioactive lipids, among those are ceramide and SPH. Under normal circumstances, in young oocytes the described pathways work just fine. But, in the old oocyte downregulation of CERT directly causes accumulation of ceramide in the ER that induces ER stress and apoptosis. Indirectly, downregulation of CERT reduces mitochondrial levels of ceramide, because the cell fails to provide sufficient $\mathrm{SPH}$, the ceramide precursor, consequently impairing MITO function and morphology. As to which response occurs first or is more important between the ER stress and the mitochondrial alterations, still remains to be demonstrated.

Despite the complex biochemistry of ceramide, our endeavor is to further understand the cellular and molecular mechanisms that maintain physiological levels of this sphingolipid in vivo in oocytes, and implications for the aging process itself. At least two possibilities may account for ceramide increases in mitochondria. First, ceramide formed in the ER can be transferred to MITO via catalyzed exchanges of ER-MITO membrane contacts (Novgorodov et al. 2011). In contrast, MITO could represent a specialized compartment of sphingolipid metabolism with their own subset of biosynthetic and degradative enzymes. A conventional view suggests that ceramide content is balanced by enzyme activities, involving both ceramide production (CerSs and SMases) and ceramide degradation (CDases; Hannun \& Obeid 2008). Intriguingly, purified neutral CDase (NCDase) catalyzes both the hydrolysis and synthesis of ceramide (Tani et al. 2000, El Bawab et al. 2001). Novgorodov et al. (2011) recently showed that NCDase is a key participant of ceramide formation in liver mitochondria. The activity of NCDase causes formation of an additional source of ceramide in mitochondria by catalyzing a reverse reaction where formation of ceramide is the result of condensation of palmitate and SPH (Novgorodov et al. 2011). On the basis of molecular cloning and confocal microscopy data, this activity was ascribed to mitochondria (El Bawab et al. 2000); and it was demonstrated in purified mitochondria (Bionda et al. 2004). A recent report suggests that ceramide could be also generated by novel mitochondrial neutral SMase hydrolyzing SM (Wu et al. 2010). Given the existence and the likelihood for each one of these various pathways to occur in the mitochondria, continued research efforts are required to better understand the mechanisms of mitochondrial ceramide generation and utilization, along with their influence on mitochondrial functions.

Because of the importance of sphingolipids as components of membranes, it is possible to speculate that reduced sphingolipid levels in mitochondria may either increase the demand on, and/or decrease the capacity of mitochondrial function by affecting mitochondrial membrane dynamics. Increased load (of either handling or processing) of defective membrane organelles could exceed the capacity of oocyte mitochondrial respiration, resulting in cellular demise. Before this final outcome, decreases in mitochondrial function might manifest as slow changes in oocyte quality. 


\section{Summary}

Thus, it seems increasingly likely that certain changes/ shifts in the metabolism of ceramide in subcellular compartments of the oocyte are associated with signaling of biochemical or morphological alterations linked to aging. Nevertheless, the downstream targets of ceramide signaling or other yet undescribed early signals activated by aging in germ cells remain to be fully elucidated. Our data point toward the involvement of ceramide in the age-dependent changes in the structure and function of oocyte mitochondria. Although most studies are often centered on searching for a unitary function of ceramide, the ensuing observations often reveal not only several functions, but also at times contradictory ones depending on cell type and other variables. Therefore, this scenario renders a unified understanding of ceramide function difficult, if not misleading. For example, in contrast to previous reports suggesting that ceramide is proapoptotic in vitro and can mediate both stress-induced intrinsic and death receptormediated extrinsic apoptosis (Taha et al. 2006, Eliyahu et al. 2007, 2010, Lahiri \& Futerman 2007), our data suggest that reduction of mitochondrial ceramide in oocytes depresses oocyte quality. Our data agree with studies where reduction of ceramide or complex sphingolipids results in progressive death of neurons, particularly Purkinje cell loss (Zhao et al. 2011). Moreover, intracerebroventricular administration of global ceramide inhibitors has been reported to cause acute neurodegeneration (Osuchowski et al. 2005). Therefore, contrary to the in vitro data, those findings, together with our results, demonstrate that decreases in ceramide synthesis can have devastating cellular consequences in vivo. Since under normal conditions sphingolipid homeostasis is critically balanced in the cell, it is not totally unexpected to find that either increases or decreases of ceramide are equally detrimental to the cell.

Alternatively, ceramide species possessing different fatty acyl chains may play distinct physiological roles. For example, loss of one of the two ceramide synthases in Caenorhabditis elegans, which produce different ceramide species, resulted in opposite outcomes under hypoxic conditions (Menuz et al. 2009). Thus, conceptualization of a unitary ceramide function is deceptive; but rather, a possible common theme is that individual molecular species of ceramide are likely regulated by specific biochemical pathways in distinct subcellular compartments, and modulate distinct functions. Therefore, endogenous ceramide production should be considered in its topological context. For example, we hypothesize that, in women, beyond a certain age, decreases of ceramide in the mitochondria, coupled with increases in the ER contribute to diminished oocyte quality.
As to what triggers these alterations, and what stoichiometric ratios are critical or are at the threshold remain unclear. But, we postulate that either transport or biosynthesis of ceramide is critical for organelle homeostasis. Thus, oocyte aging is precipitated by shifts in the synthesis and the catabolism of ceramide, in concert with changes in biochemical and structural properties of mitochondria.

This scenario reminds us that sphingolipid metabolism is highly connected and integrated. Therefore, attempts at dissecting specific pathways of ceramide metabolism and function need to consider not only ceramides but also their metabolites that may mediate their own specific actions. In any case, and despite the culprit (C16-ceramide, or CERT, or CerS6, or NCDase) responsible for decreases in oocyte quality with age, studying oocyte lipid biology opens new and exciting areas of research awaiting to be fully explored.

\section{Declaration of interest}

The authors declare that there is no conflict of interest that could be perceived as prejudicing the impartiality of the research reported.

\section{Funding}

We thank the Department of Physiology at Michigan State University, and La Junta de Andalucia in Seville Spain, for their partial financial support of this work.

\section{References}

Acton BM, Jurisicova A, Jurisica I \& Casper RF 2004 Alterations in mitochondrial membrane potential during preimplantation stages of mouse and human embryo development. Molecular Human Reproduction 10 23-32. (doi:10.1093/molehr/gah004)

Adam D, Heinrich M, Kabelitz D \& Schutze S 2002 Ceramide: does it matter for T cells? Trends in Immunology 23 1-4. (doi:10.1016/S14714906(01)02091-9)

Ames BN, Shigenaga MK \& Hagen TM 1995 Mitochondrial decay in aging. Biochimica et Biophysica Acta 1271 165-170.

Barritt JA, Brenner CA, Cohen J \& Matt DW 1999 Mitochondrial DNA rearrangements in human oocytes and embryos. Molecular Human Reproduction 5 927-933. (doi:10.1093/molehr/5.10.927)

Beemsterboer SN, Homburg R, Gorter NA, Schats R, Hompes PG \& Lambalk CB 2006 The paradox of declining fertility but increasing twinning rates with advancing maternal age. Human Reproduction $\mathbf{2 1}$ 1531-1532. (doi:10.1093/humrep/del009)

Bionda C, Portoukalian J, Schmitt D, Rodriguez-Lafrasse C \& Ardail D 2004 Subcellular compartmentalization of ceramide metabolism: MAM (mitochondria-associated membrane) and/or mitochondria? Biochemical Journal 382 527-533. (doi:10.1042/BJ20031819)

van Blitterswijk WJ, van der Luit AH, Caan W, Verheij M \& Borst J 2001 Sphingolipids related to apoptosis from the point of view of membrane structure and topology. Biochemical Society Transactions 29 819-824. (doi:10.1042/BST0290819)

Braveman FR 2006 Pregnancy in patients of advanced maternal age. Anesthesiology Clinics 24 637-646. (doi:10.1016/j.atc.2006.05.002)

Brenner CA, Wolny YM, Barritt JA, Matt DW, Munne S \& Cohen J 1998 Mitochondrial DNA deletion in human oocytes and embryos. Molecular Human Reproduction 4 887-892. (doi:10.1093/molehr/4.9.887) 
Broekmans FJ, Soules MR \& Fauser BC 2009 Ovarian aging: mechanisms and clinical consequences. Endocrine Reviews 30 465-493. (doi:10. 1210/er.2009-0006)

Clarke CJ, Wu BX \& Hannun YA 2011 The neutral sphingomyelinase family: identifying biochemical connections. Advances in Enzyme Regulation 51 51-58. (doi:10.1016/j.advenzreg.2010.09.016)

Cohen J, Scott R, Alikani M, Schimmel T, Munné S, Levron J, Wu L, Brenner C, Warner C \& Willadsen S 1998 Ooplasmic transfer in mature human oocytes. Molecular Human Reproduction 4 269-280. (doi:10. 1093/molehr/4.3.269)

Cukurcam S, Betzendahl I, Michel G, Vogt E, Hegele-Hartung C, Lindenthal B \& Eichenlaub-Ritter U 2007 Influence of follicular fluid meiosis-activating sterol on aneuploidy rate and precocious chromatid segregation in aged mouse oocytes. Human Reproduction 22 815-828. (doi:10.1093/humrep/del442)

Cummins JM 2000 Fertilization and elimination of the paternal mitochondrial genome. Human Reproduction 15 (Supplement 2) 92-101.

Cummins JM 2002 The role of maternal mitochondria during oogenesis, fertilization and embryogenesis. Reproductive Biomedicine Online 4 176-182. (doi:10.1016/S1472-6483(10)61937-2)

Cummins JM 2004a Mitochondria in reproduction. Reproductive Biomedicine Online 8 14-15. (doi:10.1016/S1472-6483(10)60493-2)

Cummins JM 2004 $b$ The role of mitochondria in the establishment of oocyte functional competence. European Journal of Obstetrics, Gynecology, and Reproductive Biology 115 (Supplement 1) S23-S29. (doi:10.1016/ j.ejogrb.2004.01.011)

Cutler RG \& Mattson MP 2001 Sphingomyelin and ceramide as regulators of development and lifespan. Mechanisms of Ageing and Development 122 895-908. (doi:10.1016/S0047-6374(01)00246-9)

Danial NN \& Korsmeyer SJ 2004 Cell death: critical control points. Cell 116 205-219. (doi:10.1016/S0092-8674(04)00046-7)

Diatlovitskaia EV, Andreasian GO \& Malykh la N 1995 Human ovarian ceramides and gangliosides in aging. Biokhimiia $601302-1306$.

D'Mello NP, Childress AM, Franklin DS, Kale SP, Pinswasdi C \& Jazwinski SM 1994 Cloning and characterization of LAG1, a longevityassurance gene in yeast. Journal of Biological Chemistry 269 15451-15459.

Dumollard R, Duchen M \& Carroll J 2007 The role of mitochondrial function in the oocyte and embryo. Current Topics in Developmental Biology 77 21-49. (doi:10.1016/S0070-2153(06)77002-8)

Eichenlaub-Ritter U, Vogt E, Yin H \& Gosden R 2004 Spindles, mitochondria and redox potential in ageing oocytes. Reproductive Biomedicine Online 8 45-58. (doi:10.1016/S1472-6483(10)60497-X)

Eichenlaub-Ritter U, Staubach N \& Trapphoff T 2010 Chromosomal and cytoplasmic context determines predisposition to maternal age-related aneuploidy: brief overview and update on MCAK in mammalian oocytes. Biochemical Society Transactions 38 1681-1686. (doi:10.1042/ BST0381681)

Eichenlaub-Ritter U, Wieczorek M, Luke S \& Seidel T 2011 Age related changes in mitochondrial function and new approaches to study redox regulation in mammalian oocytes in response to age or maturation conditions. Mitochondrion 11 783-796. (doi:10.1016/j.mito.2010.08. 011)

El Bawab S, Roddy P, Qian T, Bielawska A, Lemasters JJ \& Hannun YA 2000 Molecular cloning and characterization of a human mitochondrial ceramidase. Journal of Biological Chemistry 275 21508-21513. (doi:10. 1074/jbc.M002522200)

El Bawab S, Birbes H, Roddy P, Szulc ZM, Bielawska A \& Hannun YA 2001 Biochemical characterization of the reverse activity of rat brain ceramidase. A CoA-independent and fumonisin B1-insensitive ceramide synthase. Journal of Biological Chemistry 276 16758-16766. (doi:10. 1074/jbc.M009331200)

Eliyahu E, Park JH, Shtraizent N, He X \& Schuchman EH 2007 Acid ceramidase is a novel factor required for early embryo survival. FASEB Journal 21 1403-1409. (doi:10.1096/fj.06-7016com)

Eliyahu E, Shtraizent N, Martinuzzi K, Barritt J, He X, Wei H, Chaubal S, Copperman AB \& Schuchman EH 2010 Acid ceramidase improves the quality of oocytes and embryos and the outcome of in vitro fertilization. FASEB Journal 24 1229-1238. (doi:10.1096/fj.09-145508)

Faddy MJ 2000 Follicle dynamics during ovarian ageing. Molecular and Cellular Endocrinology 163 43-48. (doi:10.1016/S0303-7207(99) 00238-5)
Faddy MJ, Gosden RG, Gougeon A, Richardson SJ \& Nelson JF 1992 Accelerated disappearance of ovarian follicles in mid-life: implications for forecasting menopause. Human Reproduction 7 1342-1346.

Futerman AH \& Riezman H 2005 The ins and outs of sphingolipid synthesis. Trends in Cell Biology 15 312-318. (doi:10.1016/j.tcb.2005.04.006)

Gault CR, Obeid LM \& Hannun YA 2010 An overview of sphingolipid metabolism: from synthesis to breakdown. Advances in Experimental Medicine and Biology 688 1-23.

Giles RE, Blanc H, Cann HM \& Wallace DC 1980 Maternal inheritance of human mitochondrial DNA. PNAS 77 6715-6719. (doi:10.1073/pnas. 77.11.6715)

Gougeon A 2005 The biological aspects of risks of infertility due to age: the female side. Revue d'Épidémiologie et de Santé Publique 53 2S37-2S45. (doi:10.1016/S0398-7620(05)84766-2)

Guillas I, Kirchman PA, Chuard R, Pfefferli M, Jiang JC, Jazwinski SM \& Conzelmann A 2001 C26-CoA-dependent ceramide synthesis of Saccharomyces cerevisiae is operated by Lag1p and Lac1p. EMBO Journal 20 2655-2665. (doi:10.1093/emboj/20.11.2655)

Habbema JD, Eijkemans MJ, Nargund G, Beets G, Leridon H \& Te Velde ER 2009 The effect of in vitro fertilization on birth rates in western countries. Human Reproduction 24 1414-1419. (doi:10.1093/humrep/dep004)

Hanada K 2006 Discovery of the molecular machinery CERT for endoplasmic reticulum-to-Golgi trafficking of ceramide. Molecular and Cellular Biochemistry 286 23-31. (doi:10.1007/s11010-005-9044-z)

Hanada K 2010 Intracellular trafficking of ceramide by ceramide transfer protein. Proceedings of the Japan Academy. Series B, Physical and Biological Sciences 86 426-437. (doi:10.2183/pjab.86.426)

Hanada K, Kumagai K, Yasuda S, Miura Y, Kawano M, Fukasawa M \& Nishijima M 2003 Molecular machinery for non-vesicular trafficking of ceramide. Nature 426 803-809. (doi:10.1038/nature02188)

Hanada K, Kumagai K, Tomishige N \& Kawano M 2007 CERT and intracellular trafficking of ceramide. Biochimica et Biophysica Acta 1771 644-653. (doi:10.1016/j.bbalip.2007.01.009)

Hannun YA \& Obeid LM 2008 Principles of bioactive lipid signalling: lessons from sphingolipids. Nature Reviews. Molecular Cell Biology 9 139-150. (doi:10.1038/nrm2329)

Hannun YA \& Obeid LM 2011 Many ceramides. Journal of Biological Chemistry 286 27855-27862. (doi:10.1074/jbc.R111.254359)

Hardy K, Spanos S, Becker D, lannelli P, Winston RM \& Stark J 2001 From cell death to embryo arrest: mathematical models of human preimplantation embryo development. PNAS 98 1655-1660. (doi:10.1073/ pnas.98.4.1655)

Hetz CA, Hunn M, Rojas P, Torres V, Leyton L \& Quest AF 2002 Caspasedependent initiation of apoptosis and necrosis by the Fas receptor in lymphoid cells: onset of necrosis is associated with delayed ceramide increase. Journal of Cell Science 115 4671-4683. (doi:10.1242/jcs. 00153)

Hunt PA \& Hassold TJ 2008 Human female meiosis: what makes a good egg go bad? Trends in Genetics 24 86-93. (doi:10.1016/j.tig.2007.11.010)

Janny L \& Menezo YJ 1996 Maternal age effect on early human embryonic development and blastocyst formation. Molecular Reproduction and Development 45 31-37. (doi:10.1002/(SICI)1098-2795(199609)45:1 < 31::AID-MRD4 > 3.0.CO;2-T)

Jansen RP \& de Boer K 1998 The bottleneck: mitochondrial imperatives in oogenesis and ovarian follicular fate. Molecular and Cellular Endocrinology 145 81-88. (doi:10.1016/S0303-7207(98)00173-7)

Jurisicova A \& Acton BM 2004 Deadly decisions: the role of genes regulating programmed cell death in human preimplantation embryo development. Reproduction 128 281-291. (doi:10.1530/rep.1.00241)

Jurisicova A, Lee HJ, D'Estaing SG, Tilly J \& Perez GI 2006 Molecular requirements for doxorubicin-mediated death in murine oocytes. Cell Death and Differentiation 13 1466-1474. (doi:10.1038/sj.cdd.4401819)

Kawano M, Kumagai K, Nishijima M \& Hanada K 2006 Efficient trafficking of ceramide from the endoplasmic reticulum to the Golgi apparatus requires a VAMP-associated protein-interacting FFAT motif of CERT. Journal of Biological Chemistry 281 30279-30288. (doi:10.1074/jbc. M605032200)

Keefe DL, Niven-Fairchild T, Powell S \& Buradagunta S 1995 Mitochondrial deoxyribonucleic acid deletions in oocytes and reproductive aging in women. Fertility and Sterility $64577-583$.

Kirkwood TB 1998 Ovarian ageing and the general biology of senescence. Maturitas 30 105-111. (doi:10.1016/S0378-5122(98)00065-6) 
Kumagai K, Yasuda S, Okemoto K, Nishijima M, Kobayashi S \& Hanada K 2005 CERT mediates intermembrane transfer of various molecular species of ceramides. Journal of Biological Chemistry 280 6488-6495. (doi:10.1074/jbc.M409290200)

Lahiri S \& Futerman AH 2007 The metabolism and function of sphingolipids and glycosphingolipids. Cellular and Molecular Life Sciences 64 2270-2284. (doi:10.1007/s00018-007-7076-0)

Lightle SA, Oakley JI \& Nikolova-Karakashian MN 2000 Activation of sphingolipid turnover and chronic generation of ceramide and sphingosine in liver during aging. Mechanisms of Ageing and Development 120 111-125. (doi:10.1016/S0047-6374(00)00191-3)

McConnell JM \& Petrie L 2004 Mitochondrial DNA turnover occurs during preimplantation development and can be modulated by environmental factors. Reproductive Biomedicine Online 9 418-424. (doi:10.1016/ S1472-6483(10)61277-1)

Mencarelli C, Losen M, Hammels C, De Vry J, Hesselink MK, Steinbusch HW, De Baets MH \& Martinez-Martinez P 2010 The ceramide transporter and the Goodpasture antigen binding protein: one protein - one function? Journal of Neurochemistry 113 1369-1386. (doi:10.1111/j.1471-4159.2010.06673.x)

Menuz V, Howell KS, Gentina S, Epstein S, Riezman I, FornallazMulhauser M, Hengartner MO, Gomez M, Riezman H \& Martinou J-C 2009 Protection of $C$. elegans from anoxia by HYL-2 ceramide synthase. Science 324 381-384. (doi:10.1126/science.1168532)

Minami N, Suzuki T \& Tsukamoto S 2007 Zygotic gene activation and maternal factors in mammals. Journal of Reproduction and Development 53 707-715. (doi:10.1262/jrd.19029)

Novgorodov SA \& Gudz TI 2009 Ceramide and mitochondria in ischemia/reperfusion. Journal of Cardiovascular Pharmacology $\mathbf{5 3}$ 198-208. (doi:10.1097/FJC.0b013e31819b52d5)

Novgorodov SA, Wu BX, Gudz TI, Bielawski J, Ovchinnikova TV, Hannun YA \& Obeid LM 2011 Novel pathway of ceramide production in mitochondria: thioesterase and neutral ceramidase produce ceramide from sphingosine and acyl-CoA. Journal of Biological Chemistry 286 25352-25362. (doi:10.1074/jbc.M110.214866)

Okazaki T, Bielawska A, Bell RM \& Hannun YA 1990 Role of ceramide as a lipid mediator of 1 alpha,25-dihydroxyvitamin $\mathrm{D}_{3^{-}}$ induced HL-60 cell differentiation. Journal of Biological Chemistry 265 15823-15831.

Osuchowski MF, Edwards GL \& Sharma RP 2005 Fumonisin B1-induced neurodegeneration in mice after intracerebroventricular infusion is concurrent with disruption of sphingolipid metabolism and activation of proinflammatory signaling. Neurotoxicology 26 211-221. (doi:10. 1016/j.neuro.2004.10.001)

Ottolenghi C, Uda M, Hamatani T, Crisponi L, Garcia JE, Ko M, Pilia G, Sforza C, Schlessinger D \& Forabosco A 2004 Aging of oocyte, ovary, and human reproduction. Annals of the New York Academy of Sciences 1034 117-131. (doi:10.1196/annals.1335.015)

Pepe S 2005 Effect of dietary polyunsaturated fatty acids on age-related changes in cardiac mitochondrial membranes. Experimental Gerontology 40 751-758. (doi:10.1016/j.exger.2005.03.013)

Perez GI, Trbovich AM, Gosden RG \& Tilly JL 2000 Mitochondria and the death of oocytes. Nature 403 500-501. (doi:10.1038/35000651)

Perez GI, Jurisicova A, Matikainen T, Moriyama T, Kim MR, Takai Y, Pru JK, Kolesnick RN \& Tilly JL 2005 A central role for ceramide in the agerelated acceleration of apoptosis in the female germline. FASEB Journal 19 860-862.

Perheentupa A \& Huhtaniemi I 2009 Aging of the human ovary and testis. Molecular and Cellular Endocrinology 299 2-13. (doi:10.1016/j.mce. 2008.11.004)

Rao RP, Yuan C, Allegood JC, Rawat SS, Edwards MB, Wang X, Merrill AH Jr, Acharya U \& Acharya JK 2007 Ceramide transfer protein function is essential for normal oxidative stress response and lifespan. PNAS 104 11364-11369. (doi:10.1073/pnas.0705049104)

Richardson SJ, Senikas V \& Nelson JF 1987 Follicular depletion during the menopausal transition: evidence for accelerated loss and ultimate exhaustion. Journal of Clinical Endocrinology and Metabolism 65 1231-1237. (doi:10.1210/jcem-65-6-1231)

Ruman J, Klein J \& Sauer MV 2003 Understanding the effects of age on female fertility. Minerva Ginecologica 55 117-127.
Sastre J, Borras C, Garcia-Sala D, Lloret A, Pallardo FV \& Vina J 2002 Mitochondrial damage in aging and apoptosis. Annals of the New York Academy of Sciences 959 448-451. (doi:10.1111/j.1749-6632.2002. tb02114.x)

Schorling S, Vallee B, Barz WP, Riezman H \& Oesterhelt D 2001 Lag1p and Lac1p are essential for the acyl-CoA-dependent ceramide synthase reaction in Saccharomyces cerevisae. Molecular Biology of the Cell 12 $3417-3427$.

Schultz GA \& Heyner S 1992 Gene expression in pre-implantation mammalian embryos. Mutation Research 296 17-31. (doi:10.1016/ 0165-1110(92)90029-9)

Sutovsky P, Moreno RD, Ramalho-Santos J, Dominko T, Simerly C \& Schatten G 2000 Ubiquitinated sperm mitochondria, selective proteolysis, and the regulation of mitochondrial inheritance in mammalian embryos. Biology of Reproduction 63 582-590. (doi:10.1095/biolreprod63.2.582)

Taha TA, Mullen TD \& Obeid LM 2006 A house divided: ceramide, sphingosine, and sphingosine-1-phosphate in programmed cell death. Biochimica et Biophysica Acta 1758 2027-2036. (doi:10.1016/j. bbamem.2006.10.018)

Tang F, Kaneda M, O'Carroll D, Hajkova P, Barton SC, Sun YA, Lee C, Tarakhovsky A, Lao K \& Surani MA 2007 Maternal microRNAs are essential for mouse zygotic development. Genes and Development 21 644-648. (doi:10.1101/gad.418707)

Tani M, Okino N, Mitsutake S, Tanigawa T, Izu H \& Ito M 2000 Purification and characterization of a neutral ceramidase from mouse liver. A single protein catalyzes the reversible reaction in which ceramide is both hydrolyzed and synthesized. Journal of Biological Chemistry 275 3462-3468. (doi:10.1074/jbc.275.5.3462)

Tarlatzis BC \& Zepiridis L 2003 Perimenopausal conception. Annals of the New York Academy of Sciences 997 93-104. (doi:10.1196/annals.1290.011)

Thouas GA, Trounson AO, Wolvetang EJ \& Jones GM 2004 Mitochondrial dysfunction in mouse oocytes results in preimplantation embryo arrest in vitro. Biology of Reproduction 71 1936-1942. (doi:10.1095/ biolreprod.104.033589)

Thouas GA, Trounson AO \& Jones GM 2005 Effect of female age on mouse oocyte developmental competence following mitochondrial injury. Biology of Reproduction 73 366-373. (doi:10.1095/biolreprod.105.040956)

Thouas GA, Trounson AO \& Jones GM 2006 Developmental effects of sublethal mitochondrial injury in mouse oocytes. Biology of Reproduction 74 969-977. (doi:10.1095/biolreprod.105.048611)

Torres-Padilla ME, Bannister AJ, Hurd PJ, Kouzarides T \& Zernicka-Goetz M 2006 Dynamic distribution of the replacement histone variant H3.3 in the mouse oocyte and preimplantation embryos. International Journal of Developmental Biology 50 455-461. (doi:10.1387/ijdb.052073mt)

Tran D, Gascard P, Berthon B, Fukami K, Takenawa T, Giraud F \& Claret M 1993 Cellular distribution of polyphosphoinositides in rat hepatocytes. Cellular Signalling 5 565-581. (doi:10.1016/0898-6568(93)90052-N)

Tuuf J, Kjellberg MA, Molotkovsky JG, Hanada K \& Mattjus P 2011 The intermembrane ceramide transport catalyzed by CERT is sensitive to the lipid environment. Biochimica et Biophysica Acta 1808 229-235. (doi:10.1016/j.bbamem.2010.09.011)

Van Blerkom J 2004 Mitochondria in human oogenesis and preimplantation embryogenesis: engines of metabolism, ionic regulation and developmental competence. Reproduction 128 269-280. (doi:10.1530/rep.1. 00240)

Van Blerkom J 2011 Mitochondrial function in the human oocyte and embryo and their role in developmental competence. Mitochondrion 11 797-813. (doi:10.1016/j.mito.2010.09.012)

Van Blerkom J, Davis PW \& Lee J 1995 ATP content of human oocytes and developmental potential and outcome after in-vitro fertilization and embryo transfer. Human Reproduction 10 415-424.

Van Blerkom J, Sinclair J \& Davis P 1998 Mitochondrial transfer between oocytes: potential applications of mitochondrial donation and the issue of heteroplasmy. Human Reproduction 13 2857-2868. (doi:10.1093/ humrep/13.5.1317)

Van Blerkom J, Cox H \& Davis P 2006 Regulatory roles for mitochondria in the peri-implantation mouse blastocyst: possible origins and developmental significance of differential DeltaPsim. Reproduction 131 961-976. (doi:10.1530/rep.1.00458) 
Veatch JR, McMurray MA, Nelson ZW \& Gottschling DE 2009 Mitochondrial dysfunction leads to nuclear genome instability via an iron-sulfur cluster defect. Cell 137 1247-1258. (doi:10.1016/j.cell.2009.04.014)

Venable ME, Webb-Froehlich LM, Sloan EF \& Thomley JE 2006 Shift in sphingolipid metabolism leads to an accumulation of ceramide in senescence. Mechanisms of Ageing and Development 127 473-480. (doi:10.1016/j.mad.2006.01.003)

Wang X 2001 The expanding role of mitochondria in apoptosis. Genes and Development 15 2922-2933.

Wang X, Rao RP, Kosakowska-Cholody T, Masood MA, Southon E, Zhang $\mathrm{H}$, Berthet C, Nagashim K, Veenstra TK, Tessarollo L et al. 2009 Mitochondrial degeneration and not apoptosis is the primary cause of embryonic lethality in ceramide transfer protein mutant mice. Journal of Cell Biology 184 143-158. (doi:10.1083/jcb.200807176)

Wilding M, Dale B, Marino M, di Matteo L, Alviggi C, Pisaturo ML, Lombardi L \& De Placido G 2001 Mitochondrial aggregation patterns and activity in human oocytes and preimplantation embryos. Human Reproduction 16 909-917. (doi:10.1093/humrep/16.5.909)

Wu BX, Rajagopalan V, Roddy PL, Clarke CJ \& Hannun YA 2010 Identification and characterization of murine mitochondria-associated neutral sphingomyelinase (MA-nSMase), the mammalian sphingomyelin phosphodiesterase 5. Journal of Biological Chemistry 285 17993-18002. (doi:10.1074/jbc.M110.102988)
Yang Q, Gong ZJ, Zhou Y, Yuan JQ, Cheng J, Tian L, Li S, Lin XD, Xu R, Zhu ZR et al. 2010 Role of Drosophila alkaline ceramidase (Dacer) in Drosophila development and longevity. Cellular and Molecular Life Sciences 67 1477-1490. (doi:10.1007/s00018-010-0260-7)

Yoshida N, Brahmajosyula M, Shoji S, Amanai M \& Perry AC 2007 Epigenetic discrimination by mouse metaphase II oocytes mediates asymmetric chromatin remodeling independently of meiotic exit. Developmental Biology 301 464-477. (doi:10.1016/j.ydbio.2006.08.006)

Yu J, Novgorodov SA, Chudakova D, Zhu H, Bielawska A, Bielawski J, Obeid LM, Kindy MS \& Gudz TI 2007 JNK3 signaling pathway activates ceramide synthase leading to mitochondrial dysfunction. Journal of Biological Chemistry 282 25940-25949. (doi:10.1074/jbc. M701812200)

Zhao L, Spassieva SD, Jucius TJ, Shultz LD, Shick HE, Macklin WB, Hannun YA, Obeid LM \& Ackerman SL 2011 A deficiency of ceramide biosynthesis causes cerebellar purkinje cell neurodegeneration and lipofuscin accumulation. PLoS Genetics 7 e1002063. (doi:10.1371/ journal.pgen.1002063)

Received 18 September 2011

First decision 18 October 2011

Accepted 31 October 2011 